\title{
Research on multi-stage iterative model solving method with resource optimization configuration
}

\author{
Qihua Tian ${ }^{1}$, Yurong Zhang ${ }^{1, *}$, Qunmei Dong ${ }^{2}$, Xiangman Zhou ${ }^{1}$ and Yixian $\mathrm{Du}^{1}$ \\ ${ }^{1}$ College of Mechanical and Power Engineering, China Three Gorges University, Yichang 443002, \\ China \\ ${ }^{2}$ Guodian Dadu River Repair \& Installation Co., Ltd., Leshan 614000, China
}

Keywords: Multi-stage iterative model, Resource constraints, Resource optimization configuration, Optimum operating efficiency, Time cost.

\begin{abstract}
Aiming at the problem of unreasonable resource allocation in the existing coupling design iterative model based on efficiency constraint, the resource equilibrium strategy was introduced into the iterative model. In order to reduce the time cost of product development, a multi-stage iterative model with optimal resource allocation was constructed and the optimal resource allocation of each task group was obtained by solving this model. Taking the design and development process of an air purifier as an example, the validity of this model was verified. The research shows that after introducing resource equilibrium strategy into the iterative model based on efficiency constraint, the time cost of iterative mode in different stages decreases, and with the increase of the number of iteration stages, the time cost decreases first and then increases. The research results provide a theoretical basis for designers to reasonably select resource allocation mode and number of iteration stages in actual product development so as to optimize resource allocation and reduce development cost.
\end{abstract}

\section{Introduction}

The design and development of products is a key link in the competition and development of modern enterprises. The process generally shows the coupling and iteration of complexity, especially the design and development of complex products. Because the product design and development process will be constrained by resources from the internal personnel, equipment, funds, etc., and the unreasonable distribution of these resources will directly lead to the extension of the product development cycle. Therefore, aiming at the coupling problem in product design, the appropriate iterative solution method is proposed to realize the reasonable allocation of design and development resources, which is of great concern to people. At present, some scholars have studied this problem from different aspects. For example, Sun Xiaobin et al. measured the critical degree of different tasks in parallel iteration process by introducing the optimal operational efficiency value, and established an optimization model of parallel iteration based on efficiency constraints. By optimizing the solution, they got the optimal operational efficiency value and critical degree of different task groups under a

*Corresponding author: 1803952584@qq..com 
specific design structure, and provided technology for the optimal state of resource allocation level of each task group. Kim D. introduced the work transformation matrix into the research of parallel iterative design, and developed a systematic representation of the work transformation matrix, but these studies are based on parallel iterative design. Xiao Renbin et al. solved and analyzed the resource optimization problem of phased iteration model from the aspects of task iteration time and the validity of iteration times, combining with genetic algorithm. However, this method is mainly proposed for the iteration process of specified phases, and the research on the phased iteration process with uncertain phases needs further study. Tian Qihua et al. [4,5] respectively studied the coupling set solution model and the two-stage iteration model solution method with the characteristics of resource optimal allocation, but they do not take into account the multi-stage iterative mode above the second order. Based on the existing iterative model based on efficiency constraints, this paper introduced the resource equilibrium configuration strategy into the parallel iterative model, and constructed a multi-stage iterative solution model based on efficiency constraints with resource optimization configuration, in order to realize the optimal allocation of resources in the process of product development, thus achieve the goal of shortening the product development cycle.

\section{Multi-stage iterative model analysis based on efficiency constraints}

The multi-stage iteration model mainly divides the parallel iteration into two or more stages, and all tasks in each stage implement the parallel iteration, while the serial iteration is implemented between each stage. Reference Wu Weijie. [6], the design structure matrix (DSM) is used to construct the multi-stage iteration model. Quantitative DSM diagonal elements describe the length of time required for all tasks to execute independently, while non-diagonal elements represent the possibility of corresponding tasks occurring, and its value is in $[0,1]$. Each non-diagonal unit value represents the probability of another iteration process occurring when task $\mathrm{i}$ does not receive the latest output information from task $\mathrm{j}$.

Defining a diagonal matrix:

$$
V=\operatorname{diag}\left(v_{1}, v_{2}, \cdots, v_{i}, \cdots, v_{n}\right)
$$

The elements $v_{i}$ on the diagonal line are the efficiency of the corresponding working group to complete the task $i$, and $\mathrm{n}$ is the total number of tasks in the product design and development process. The capital inflow, manpower input, technical conditions and other resources of each design task group are allocated by enterprises based on past design experience. After the resources of the coupled centralized reserve task group are allocated quantitatively, their corresponding operational efficiency $v_{i}$ has its own upper limit max $v_{i}$, which results in the upper limit constraints of the reserve task group $v_{i}$, the constraints are: 0 $<v_{i} \leq \max v_{i}$. Let the execution time of each stage be $T_{1}, T_{2} \ldots, T_{s}, \ldots, T_{m}$ are all n-dimensional column vectors, and $m$ is the total number of iteration stages $(s=1,2, \ldots, m, m \leq n)$, where the execution time of stage $s$ is:

$$
\boldsymbol{T}_{s}=\left[\begin{array}{llllll}
t_{s 1} & t_{s 2} & \cdots & t_{s j} & \cdots & t_{s n}
\end{array}\right]^{T}
$$

In the Eq. $2, t_{s j}$ represents the execution time of the $j$ task in the current s stage $(j=1,2, \ldots$, $n$ ), and the maximum number of tasks in phase $s$ is $n$.

According to reference Chen Tinggui et al. [7], the execution time of the first stage of the multi-stage iterative model is as follows: 


$$
\boldsymbol{T}_{1}=\boldsymbol{Z}\left(\boldsymbol{I}-\boldsymbol{K}_{1} \boldsymbol{R} \boldsymbol{K}_{1}\right)^{-1} \boldsymbol{K}_{1} \boldsymbol{u}_{0}
$$

In the formula, $Z$ is the task cycle matrix, which is composed of the elements on the diagonal of the DSM and contains the execution cycle of each task; $R$ is the rework probability matrix, which is composed of the elements on the non-diagonal elements of DSM and represents the iteration process. The initial work vector $\mathrm{u}_{0}$ is all 1 vector, indicating that all tasks in the first iteration need to be executed at the same time; $I$ is the unit matrix. Matrix $K_{i}$ is one-stage task distribution matrix, which describes the performance of tasks in the first stage of iteration. The value of the element is defined as follows:

$$
\begin{gathered}
k_{1}^{i j}= \begin{cases}1 & \text { if } i=j \text { and task } i \text { is in the first stage } \\
0 & \text { others }\end{cases} \\
\boldsymbol{T}_{2}=\boldsymbol{Z}\left(\boldsymbol{I}-\boldsymbol{K}_{2} \boldsymbol{R} \boldsymbol{K}_{2}\right)^{-1}\left(\boldsymbol{K}_{2}-\boldsymbol{K}_{1}\right) \boldsymbol{u}_{0} \\
k_{s}^{i j}= \begin{cases}1 & \text { if } i=j \text { and task } i \text { is in } 1,2 \text { stage } \\
0 & \text { others }\end{cases}
\end{gathered}
$$

The execution time $T_{s}$ required for stage $s$ is:

$$
\boldsymbol{T}_{s}=\boldsymbol{Z}\left(\boldsymbol{I}-\boldsymbol{K}_{s} \boldsymbol{R} \boldsymbol{K}_{s}\right)^{-1}\left(\boldsymbol{K}_{s}-\boldsymbol{K}_{s-1}\right) \boldsymbol{u}_{0}
$$

In the formula, matrix $K_{s}$ is the task distribution matrix of $s$ stage, which describes the execution of tasks in the iteration process of $s$ stage. The values of the element are defined as follows:

$$
k_{s}^{i j}= \begin{cases}1 & \text { if } i=j \text { and task } i \text { is in } 1,2, \cdots, s, \cdots, n \text { stage } \\ 0 & \text { others }\end{cases}
$$

By summing up the execution time of all stages, the time cost $U$ of the iteration process of the coupling set can be obtained,

$$
\boldsymbol{U}=\boldsymbol{V}^{-1} \sum_{s=1}^{m} \boldsymbol{T}_{s}=\boldsymbol{V}^{-1} \sum_{s=1}^{m}\left(\boldsymbol{Z}\left(\boldsymbol{I}-\boldsymbol{K}_{s} \boldsymbol{R} \boldsymbol{K}_{s}\right)^{-1}\left(\boldsymbol{K}_{s}-\boldsymbol{K}_{s-1}\right) \boldsymbol{u}_{0}\right)
$$

When establishing the mathematical optimization model of the iteration process, the objective of optimization should be to minimize the operation time of the task with the largest actual working time. In order to get the optimal solution, we need to take $V$ as the design variable and the total execution time $U$ as the optimization objective. The maximum operator $\overline{\max }(a)=\max \left(a_{1}, a_{2}, \cdots, a_{n}\right)$ is defined for column vector elements, where a is an $\mathrm{n}$ dimensional column vector and $a_{i}(i=1,2, \ldots, n)$ is a column vector element. Combining with the solution formula of execution time of multi-stage iteration model Eq.6, a multi-stage iteration resource allocation model based on efficiency constraints can be obtained:

$$
\left\{\begin{array}{l}
\text { find }: \boldsymbol{T}_{s}=\left[\begin{array}{llllll}
\boldsymbol{t}_{\boldsymbol{s} 1} & \boldsymbol{t}_{\boldsymbol{s} 2} & \ldots & \boldsymbol{t}_{\boldsymbol{s}} & \ldots & \boldsymbol{t}_{\boldsymbol{s} n}
\end{array}\right]^{\boldsymbol{T}} \\
\text { object }: \boldsymbol{F}=\min \left(\overline{\max }\left(\boldsymbol{V}^{-1}\left(\sum_{s=1}^{m} \boldsymbol{Z}\left(\boldsymbol{I}-\boldsymbol{K}_{s} \boldsymbol{R} \boldsymbol{K}_{s}\right)^{-1}\left(\boldsymbol{K}_{s}-\boldsymbol{K}_{s-1}\right) \boldsymbol{u}_{0}\right)\right)\right) \\
\text { s.t. }: 0<\boldsymbol{v}_{\boldsymbol{i}} \leq \max \boldsymbol{v}_{\boldsymbol{i}}, \quad \boldsymbol{s}=1,2,3, \cdots, \boldsymbol{i}, \cdots, \boldsymbol{n}
\end{array}\right.
$$

Through the above mathematical optimization model, the final optimal operating efficiency of each task group can be obtained, recorded as optimal_ $v_{i}$. Defines the critical 
degree parameter ratio_ $v_{i}$, which is:

$$
\text { ratio } v_{i}=\frac{\text { optimal } v_{i}}{\max v_{i}}
$$

This value represents the resource utilization of each task group and ranges from 0 to 1 . If the value is close to 1 , it indicates that the task team corresponding to the design task occupies more resources in its work, and the task team has more pressure in the process of performing the task; if the value is close to 0 , it indicates that the task team corresponding to the design task has the problem of waste of resources in its work, and should reduce the resource allocation of the task team and allocate more resources to other tasks execution to improve resource utilization.

\section{Construction of multi-stage iteration model with resource balancing strategy}

According to Eq.7 and Eq.8, the optimal operating efficiency optimal_ $v_{i}$ of each task group can be calculated under the existing resource allocation. The rationality of resource allocation based on the optimization model can be analyzed by calculating the critical degree ratio_ $v_{i}$ of each task group. If the resource allocation is unreasonable, a new optimization model needs to be built. However, it is difficult to optimize resource allocation based on the design experience of enterprises.

The optimization model based on efficiency constraints can optimize the time cost of task execution through resource balanced allocation (e.g., Xiao Renbin et al. [3]). The maximum operational efficiency of each task team is regarded as the amount of resources allocated to it. In order to make a reasonable redistribution of resources, the maximum operational efficiency value $\max v_{i}$ of each task group was firstly accumulated to obtain the maximum overall operational efficiency: $\sum_{i=1}^{n} \max v_{i}$. And set constraints: the operation efficiency $v_{i}$ of each task team must be less than the maximum total operation efficiency value $\sum_{i=1}^{n} \max v_{i}$; and the sum of the operation efficiency $\sum_{i=1}^{n} v_{i}$ of each task team must also be less than the maximum total operation efficiency value $\sum_{i=1}^{n} \max v_{i}$. This leads to new constraints:

Therefore, the model for introducing a resource balancing strategy with resource optimization configuration is:

From the above model analysis, it can be seen that the multi-stage iteration model with resource balancing strategy is mainly based on the optimization model based on efficiency constraints, which accumulates the maximum efficiency value of the each task, and then improves the constraints according to the cumulative efficiency, and finally obtains the optimization model.

$$
\left\{\begin{array}{l}
0<\boldsymbol{v}_{\boldsymbol{i}} \leq \sum_{i=1}^{n} \max \boldsymbol{v}_{\boldsymbol{i}} \\
0<\sum_{i=1}^{n} \boldsymbol{v}_{\boldsymbol{i}} \leq \sum_{i=1}^{m} \max \boldsymbol{v}_{\boldsymbol{i}}
\end{array}\right.
$$




$$
\left\{\begin{array}{l}
\text { find: } \boldsymbol{T}_{\boldsymbol{s}}=\left[\begin{array}{llllll}
\boldsymbol{t}_{\boldsymbol{s} 1} & \boldsymbol{t}_{\boldsymbol{s} 2} & \ldots & \boldsymbol{t}_{s j} & \ldots & \boldsymbol{t}_{\boldsymbol{s} \boldsymbol{n}}
\end{array}\right]^{\boldsymbol{T}} \\
\text { object: } \boldsymbol{F}=\min \left(\overline{\max }\left(\boldsymbol{V}^{-1}\left(\sum_{\boldsymbol{s}=1}^{\boldsymbol{m}} \boldsymbol{Z}\left(\boldsymbol{I}-\boldsymbol{K}_{\boldsymbol{m}} \boldsymbol{R} \boldsymbol{K}_{\boldsymbol{m}}\right)^{-1}\left(\boldsymbol{K}_{\boldsymbol{m}}-\boldsymbol{K}_{\boldsymbol{m}-1}\right) \boldsymbol{u}_{0}\right)\right)\right) \\
\text { s.t.: } 0<\boldsymbol{v}_{\boldsymbol{i}} \leq \sum_{i=1}^{n} \max \boldsymbol{v}_{\boldsymbol{i}}, \quad 0<\sum_{i=1}^{n} \boldsymbol{v}_{\boldsymbol{i}} \leq \sum_{i=1}^{n} \max \boldsymbol{v}_{\boldsymbol{i}}
\end{array}\right.
$$

\section{Case analysis}

\subsection{Problem description}

The development process of an air purifier is illustrated as an example. The design and development of the air purifier includes eight tasks[8]: conceptual design (Task A), fan design (Task B), air filter design (Task C), water tank design (Task D), intelligent monitoring system design (Task E), main body design (Task F), negative ion generator design (Task G) and circuit design (Task H), among them, task B, task C, task D, task E, task F and other five tasks constitute a coupled set with cyclic information flow. The design structure matrix (DSM) corresponding to the development and design task of the air purifier is shown in Fig. 1 (a). Elements on the off-diagonal of the DSM constitute the rework probability matrix R, as shown in Fig. 1 (b), which describes the numerical value of the rework amount of the task in the iterative process. For example, the value in the second column of the fifth row in figure 1 can be described as follows: $30 \%$ of the work of task F needs to be done again after task C is completed. The elements on the diagonal of the DSM constitute the task period matrix Z, as shown in Fig. 1 (c), which contains the time cycle required for each task independent execution. For example, the value of the second row and second column in figure 1 indicates that the execution cycle of task $\mathrm{c}$ is 4 .

$$
\begin{aligned}
& B \\
& B \\
& C \\
& D \\
& E \\
& F
\end{aligned}\left[\begin{array}{ccccc}
7 & 0 & 0 & 0 & 0.7 \\
0.6 & 4 & 0 & 0.6 & 0 \\
0.2 & 0 & 5 & 0.5 & 0 \\
0.5 & 0 & 0.6 & 6 & 0 \\
0.2 & 0.3 & 0 & 0 & 2
\end{array}\right] ; \quad \boldsymbol{R}=\left[\begin{array}{lllll}
0 & 0 & 0 & 0 & 0.7 \\
0.6 & 0 & 0 & 0.6 & 0 \\
0.2 & 0 & 0 & 0.5 & 0 \\
0.5 & 0 & 0.6 & 0 & 0 \\
0.2 & 0.3 & 0 & 0 & 0
\end{array}\right] ; \quad \boldsymbol{Z}=\left[\begin{array}{llll}
7 & & & \\
& 4 & & \\
& 5 & \\
& & 6 \\
& & & 2
\end{array}\right]
$$

(a) Design structure matrix (b) Rework probability matrix (c) Task period matrix

Fig. 1. Design structure matrix and its composition.

The resource allocation of the air purifier in the design process is based on previous design experience. Fixed resource allocation determines that the execution efficiency of the task teams corresponding to the five tasks has the following upper and lower limits:

$$
0<v_{1} \leq 12,0<v_{2} \leq 14,0<v_{3} \leq 10,0<v_{4} \leq 7,0<v_{5} \leq 15 \text {, that : } V=\operatorname{diag}(12,14,10,7,15)
$$

In this paper, the dynamic programming method is used to optimize the optimal task distribution schemes in different stages, and the specific process reference Moustafa Elshafei et al. [9]. According to the optimal task distribution in each stage, the definition of task distribution matrix $\mathrm{Ki}$ in Eq.3 and the calculation method of total time labor in Eq. 3 to Eq.5 are combined. A symbol encoding method is used to describe the different stages of different tasks with a numeric sequence table. The values of each coding bit represent the stages of corresponding tasks respectively. The length of the sequence table represents the number of

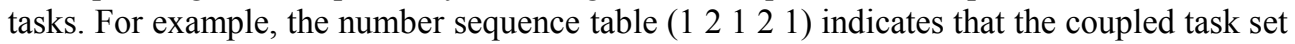
consisting of five tasks of tasks B, C, D, E, and F of this example constitutes a task 
distribution scheme of the two-stage iterative model, that is, tasks B, D, F is executed in the second phase, and tasks $\mathrm{C}$ and $\mathrm{E}$ are executed in the first phase.

\subsection{Method based on efficiency constraints}

According to Eq.7, based on the design experience, the constraints in the optimization model are the upper limit of work efficiency allocated by the reserve task team, that is,

$$
\text { s. t. } 0<\mathrm{v} 1 \leq 12,0<\mathrm{v} 2 \leq 14,0<\mathrm{v} 3 \leq 10,0<\mathrm{v} 4 \leq 7,0<\mathrm{v} 5 \leq 15
$$

The optimization objective of the model is to minimize the execution time of the design task team with the largest time cost value of the iteration process under the specified iteration mode. Since there are five design tasks in the development process of the air purifier, there are five possible iteration modes, namely one-stage iteration, two-stage iteration, three-stage iteration, four-stage iteration and five-stage iteration. One-stage iteration is also called singlestage n-stage iteration, and five-stage iteration is also called sequential iteration. The objective function of optimization under various iterations is as follows:

$$
\begin{aligned}
& \boldsymbol{F}^{(1)}=\min \left(\max \left[24.7204 / \boldsymbol{v}_{1} 25.4682 / \boldsymbol{v}_{2} 22.0655 / \boldsymbol{v}_{3} \quad 32.4816 / \boldsymbol{v}_{4} \quad 7.2328 / \boldsymbol{v}_{5}\right]\right) \\
& \boldsymbol{F}^{(2)}=\min \left(\max \left[18.2235 / \boldsymbol{v}_{1} 10.2644 / \boldsymbol{v}_{2} 12.5623 / \boldsymbol{v}_{3} 11.9014 / \boldsymbol{v}_{4} 4.5810 / \boldsymbol{v}_{5}\right]\right) \\
& \boldsymbol{F}^{(3)}=\min \left(\max \left[16.1990 / \boldsymbol{v}_{1} 5.5270 / \boldsymbol{v}_{2} 11.6327 / \boldsymbol{v}_{3} \quad 10.3645 / \boldsymbol{v}_{4} \quad 3.7547 / \boldsymbol{v}_{5}\right]\right) \\
& \boldsymbol{F}^{(4)}=\min \left(\max \left[17.2426 / \boldsymbol{v}_{1} \quad 7.9691 / \boldsymbol{v}_{2} \quad 7.7006 / \boldsymbol{v}_{3} \quad 13.0039 / \boldsymbol{v}_{4} 4.1807 / \boldsymbol{v}_{5}\right]\right) \\
& \boldsymbol{F}^{(5)}=\min \left(\max \left[14.8728 / \boldsymbol{v}_{1} 11.1471 / \boldsymbol{v}_{2} 10.7579 / \boldsymbol{v}_{3} 17.1197 / \boldsymbol{v}_{4} \quad 3.2134 / \boldsymbol{v}_{5}\right]\right)
\end{aligned}
$$

Optimal_ $k_{i}$ is used to express the optimal task distribution and optimal_ $v_{i}$ is used to express

\begin{tabular}{|c|c|c|c|c|c|c|c|}
\hline $\begin{array}{c}\text { Iterative } \\
\text { model }\end{array}$ & $i$ & B & $\mathrm{C}$ & $\mathrm{D}$ & $\mathrm{E}$ & $\mathrm{F}$ & T(Days) \\
\hline $\begin{array}{c}\text { One-stage } \\
\text { model }\end{array}$ & $\begin{array}{l}\text { optimal_k } k_{i} \\
\text { optimal_vi } \\
\text { ratio_v } v_{i}(\%)\end{array}$ & $\begin{array}{c}1 \\
5.3337 \\
24.1276\end{array}$ & $\begin{array}{c}1 \\
5.4909 \\
39.2207\end{array}$ & $\begin{array}{c}1 \\
5.4065 \\
54.0650\end{array}$ & $\begin{array}{c}1 \\
7.0000 \\
100\end{array}$ & $\begin{array}{c}1 \\
14.9925 \\
99.95\end{array}$ & 18.4770 \\
\hline $\begin{array}{c}\text { Three-stage } \\
\text { model }\end{array}$ & $\begin{array}{l}\text { optimal_k } k_{i} \\
\text { optimal_vi } \\
\text { ratio_v } v_{i}(\%)\end{array}$ & $\begin{array}{c}1 \\
12.000 \\
100\end{array}$ & $\begin{array}{c}3 \\
11.9456 \\
85.3257\end{array}$ & $\begin{array}{c}1 \\
7.9820 \\
79.820\end{array}$ & $\begin{array}{c}2 \\
7.000 \\
100\end{array}$ & $\begin{array}{c}1 \\
14.9226 \\
99.484\end{array}$ & 5.002 \\
\hline $\begin{array}{c}\text { Four-stage } \\
\text { model }\end{array}$ & $\begin{array}{l}\text { optimal_k } k_{i} \\
\text { optimal_v } v_{i} \\
\text { ratio_v } v_{i}(\%)\end{array}$ & $\begin{array}{c}1 \\
9.2975 \\
77.4791\end{array}$ & $\begin{array}{c}3 \\
8.8112 \\
62.9371\end{array}$ & $\begin{array}{c}4 \\
9.1111 \\
91.1100\end{array}$ & $\begin{array}{c}2 \\
7.000 \\
100\end{array}$ & $\begin{array}{c}1 \\
14.9562 \\
99.7080\end{array}$ & 5.7414 \\
\hline $\begin{array}{c}\text { Five-stage } \\
\text { model }\end{array}$ & $\begin{array}{l}\text { optimal_k } k_{i} \\
\text { optimal_v } v_{i} \\
\text { ratio_v } v_{i}(\%)\end{array}$ & $\begin{array}{c}1 \\
6.0971 \\
50.8092\end{array}$ & $\begin{array}{c}3 \\
4.8128 \\
34.3771\end{array}$ & $\begin{array}{c}4 \\
4.9794 \\
49.794\end{array}$ & $\begin{array}{c}2 \\
7.0000 \\
100\end{array}$ & $\begin{array}{c}5 \\
14.9854 \\
99.9026\end{array}$ & 9.5706 \\
\hline
\end{tabular}
the optimal operation efficiency and ratio_ $v_{i}$ is used to express the critical degree. With the application of MATLAB software, the optimal operating efficiency optimal_vi $v_{i}$ of each task group under each iteration stage based on efficiency constraints can be obtained, as shown in Table 1.

Table 1. Resource allocation of task teams based on efficiency constraints. 
As can be seen from table 1, in all iterations of different stages, the critical degrees of task group $\mathrm{E}$ are $100 \%$, indicating that the corresponding task group has the highest utilization rate of resources, indicating that it is in shortage of resources and has the highest working pressure. In addition, the critical degrees of task group $\mathrm{F}$ is close to $\mathrm{E}$, explain its working pressure is also high. However, the critical degrees of task group B in the one-stage iteration and task group $\mathrm{C}$ in the five-stage iteration were $24.1276 \%$ and $34.3771 \%$, respectively. The low critical degrees indicated that the design capability of these task groups was excessive, that is, there was a problem of resource waste. Task group D in one-stage iteration and task group C in two-stage iteration, their critical degrees are $54.0650 \%$ and $55.5264 \%$ respectively. Their critical degrees are moderate, which indicates that the corresponding task group has a good resource allocation. At the same time, it can be seen from Table 1. that when the number of phases of the task is not too large (the maximum number of phases in this case is 5), the time cost of the coupling set is obviously less than that of the single-stage mode, but with the increase of the number of phases, the time cost decreases first and then increases. In this case, the time cost of the three-stage iteration model is the smallest.

\subsection{Resource reorganization by introducing resource equilibrium allocation method}

According to the data analysis, it can be seen that in the iteration process of each stage, the execution process of some tasks in the iteration model of each stage has the phenomenon of waste of resources and shortage of resources, that is to say, the problem of uneven allocation of resources exists in varying degrees, so it should be adjusted appropriately. Based on the above analysis results, the five task teams can now be considered as a whole, assuming that the resources can be adjusted at will, and their overall maximum performance is 58 . The new constraint is:

s. t. $0<\mathrm{v} 1 \leqslant 58,0<\mathrm{v} 2 \leqslant 58,0<\mathrm{v} 3 \leqslant 58,0<\mathrm{v} 4 \leqslant 58,0<\mathrm{v} 5 \leqslant 58$

$$
0<\mathrm{v} 1+\mathrm{v} 2+\mathrm{v} 3+\mathrm{v} 4+\mathrm{v} 5 \leqslant 58
$$

According to the construction method of the optimization model based on resource optimal allocation in the section 2, and on the basis of the optimization model based on efficiency constraints, the constraints are improved according to the resource balancing strategy. The optimization model with resource optimal allocation is obtained as follows:

$$
\begin{aligned}
& \text { find: } \boldsymbol{T}_{s}=\left[\begin{array}{llllll}
t_{s 1} & t_{s 2} & \ldots & t_{s j} & \ldots & t_{s n}
\end{array}\right]^{T} \\
& \text { object: } \boldsymbol{F}=\min \left(\overline{\max }\left(\boldsymbol{V}^{-1}\left(\sum_{s=1}^{\boldsymbol{m}} \boldsymbol{Z}\left(\boldsymbol{I}-\boldsymbol{K}_{\boldsymbol{s}} \boldsymbol{R} \boldsymbol{K}_{\boldsymbol{s}}\right)^{-1}\left(\boldsymbol{K}_{\boldsymbol{s}}-\boldsymbol{K}_{s-1}\right) \boldsymbol{u}_{0}\right)\right)\right) \\
& \text { s.t.: } 0<\boldsymbol{v}_{1} \leq 58 \quad 0<\boldsymbol{v}_{2} \leq 58 \quad 0<\boldsymbol{v}_{3} \leq 58 \quad 0<\boldsymbol{v}_{4} \leq 58 \quad 0<\boldsymbol{v}_{5} \leq 58 \\
& 0<\boldsymbol{v}_{1}+\boldsymbol{v}_{2}+\boldsymbol{v}_{3}+\boldsymbol{v}_{4}+\boldsymbol{v}_{5} \leq 58
\end{aligned}
$$

The optimization model of Eq.12 is used to calculate the optimization model through MATLAB software, and the optimization results of the improved model are shown in Table 2:

As can be seen from Table 2, based on the method of optimizing resource allocation, the operational efficiency of each task is adjusted. For example, for the first-order iteration process, The operational effectiveness of each task group is adjusted from 12, 14, 10, 7 and 
15 to $12.8652,13.1926,11.4300,16.8256$ and 3.7446 , respectively. The resources of each working group have been reallocated, and the trend of time cost is consistent with the efficiency constraint method.

Table 2. Resource redistribution of task teams based on resource balancing strategy.

\begin{tabular}{|c|c|c|c|c|c|c|c|}
\hline \multirow{2}{*}{$\begin{array}{c}\text { Iterative } \\
\text { model }\end{array}$} & $i$ & B & $\mathrm{C}$ & $\mathrm{D}$ & E & $\mathrm{F}$ & \multirow[t]{2}{*}{$\begin{array}{c}\mathrm{T} \\
\text { (Days) }\end{array}$} \\
\hline & $\max \boldsymbol{v}_{i}$ & 12 & 14 & 10 & 7 & 15 & \\
\hline \multirow{2}{*}{$\begin{array}{l}\text { One-stage } \\
\text { model }\end{array}$} & optimal_k $k_{i}$ & 1 & 1 & 1 & 1 & 1 & \multirow{2}{*}{9.6525} \\
\hline & optimal_v $v_{i}$ & 12.8652 & 13.1926 & 11.4300 & 16.8256 & 3.7446 & \\
\hline \multirow{2}{*}{$\begin{array}{c}\text { Two-stage } \\
\text { model }\end{array}$} & optimal_ki & 1 & 2 & 1 & 2 & 1 & \multirow{2}{*}{4.9597} \\
\hline & optimal_v $v_{i}$ & 18.3715 & 10.3478 & 12.6644 & 11.9981 & 4.6182 & \\
\hline \multirow{2}{*}{$\begin{array}{l}\text { Three- } \\
\text { stage } \\
\text { model }\end{array}$} & optimal_k $k_{i}$ & 1 & 3 & 1 & 2 & 1 & \multirow{2}{*}{4.0929} \\
\hline & optimal_v $v_{i}$ & 19.7890 & 6.7519 & 14.2108 & 12.6615 & 4.5866 & \\
\hline \multirow{2}{*}{$\begin{array}{c}\text { Four-stage } \\
\text { model }\end{array}$} & optimal_k $k_{i}$ & 1 & 3 & 4 & 2 & 1 & \multirow{2}{*}{4.3187} \\
\hline & optimal_v $v_{i}$ & 19.9627 & 9.2263 & 8.9154 & 15.0553 & 4.8402 & \\
\hline \multirow{2}{*}{$\begin{array}{c}\text { Five-stage } \\
\text { model }\end{array}$} & optimal_ki & 1 & 3 & 4 & 2 & 5 & \multirow{2}{*}{4.9234} \\
\hline & optimal_v $v_{i}$ & 15.1043 & 11.3206 & 10.9254 & 17.3862 & 3.2634 & \\
\hline
\end{tabular}

Under five different iterative methods of different stages, the time cost obtained from the optimized model before and after improvement is compared, as shown in Fig. 2. It can be seen that for the first-order iterative process, the time cost of the air purifier obtained by the optimization model based on efficiency constraints is 18.4770 days, and the time cost of the air purifier obtained by the optimization model after the introduction of resource balance strategy is 9.6525 days, the latter decreased by 8.8254 days compared with the former, a decrease of $47.7594 \%$. Similarly, the time cost of the second to fifth stages decreased by $24.74 \%, 18.17 \%, 24.78 \%$, and $48.59 \%$, respectively. It can be seen that after resource restructuring based on the optimal allocation of resources, the time cost of each stage is reduced to different degrees compared with the method before improvement, which indicates the effectiveness of the optimal allocation of resources. In addition, the time cost of different stages is different. From the first stage to the fifth stage, there is a trend of first high, then low and then rising, among which three stages are the lowest.

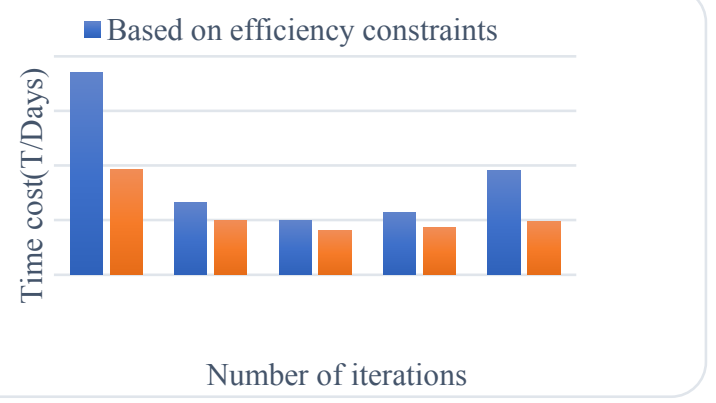

Fig. 2. Time cost based on efficiency constraint method and introduction of resource balancing strategy.

\section{Conclusions}

Based on the efficiency constraint, this paper proposes a multi-stage iterative model with resource optimization configuration. By solving the model, the optimal task allocation and 
resource allocation under multi-stage iteration are obtained. The theoretical and case analysis results show that:

(1) After introducing the resource balancing strategy on the iterative model based on efficiency constraints, the time cost in the iterative mode of different phases is lower than that of the original iterative model, indicating that the introduction of resource balancing strategy is effective;

(2) As the number of iterations increases, the time cost required to complete the task decreases first and then increases. Having more the number of iteration does not necessarily mean that it is better. In the actual product development, The designers can reasonably select the number of iteration stages according to the calculation and analysis of the model, the enterprise's own needs and the actual situation.

The multi-stage iterative optimization model with resource optimization configuration proposed in this paper can further reduce the time cost of product design and development, and provide a certain guiding basis for the design and development of actual product.

This research was financially supported by the National Natural Science Foundation of China (51475265) and the Science Foundation of China Three Gorges University (Z2019208).

\section{References}

1. Sun Xiaobin, Xiao Renbin. A Research on Concurrent Design Based On Teamwork Efficiency[J]. Journal of Huazhong University of Science and Technology(Natural Science Edition). 1997(12): 50-52.

2. Kim D. On representations and dynamic analysis of concurrent engineering design $[\mathrm{J}]$. Journal of Engineering Design, 2007, 18(3): 265-277.

3. Xiao Renbin, Zhou Rui, Chen Tinggui. Research on Solving Approach to Coupled Task Set Based on Balanced Resource Strategy [C]. The Chinese Congress of Systems Science and Systems Engineering. 2008.

4. Tian Qihua, Wang Tao, Du Yixian, Wang Weiwei, Wen Xiaoyong. Research of coupled set solving model with resource optimization configuration properties [J]. Journal of Machine Design, 2016(4): 67-71.

5. Tian Qihua, Wang Tao, Du Yixian, Dong Qunmei, Wang Cheng. Research on two-stage iterative model solving method with resource optimization configuration [J]. Journal of Machine Design, 2017,34(11): 57-62.

6. Wu Weijie. The Research of T Company Product Development Process Optimizing Based on Design Structure Matrix(DSM) [D]. South China University of Technology, 2017.

7. Chen Tinggui, Xiao Renbin. Coupled task set solving method based on inner iteration [J]. Computer Integrated Manufacturing Systems, 2008,14(12): 2375-2383.

8. Su Meixian. Research and Design of Air Purifier [D]. A Dissertation Submitted to Guangdong University of Technology for the Degree of Master, 2014.

9. Moustafa Elshafei, Hesham K. Alfares. A dynamic programming algorithm for days-off scheduling with sequence dependent labor costs [J]. Journal of Scheduling, 2008,11(2).

10. Chen Weiming. Project Scheduling Problem for Product Development under Dynamic Environment and its Solution [D]. Huazhong University of Science and Technology, 2011.

11. Xu Xiaogang. Research of DSM And It's Application to Design Management [D]. Ph.D. Dissertation of Chongqing University, 2002. 
12. Chen Ping, Yang Wenyu. Modular Clustering of Complex Product Structure and Quantity of Design Iteration Calculation [J]. China Mechanical Engineering, 2007, 18(11): 1346-1349.

13. Yang Qing, LÜ Jiarui, KHERBACHI Sonia. Progress in modeling and optimization for complex R\&D project based on design structure matrix [J]. Systems Engineering-theory \& Practice, 2016,(04): 989-1002.

14. Zhao Lihui, Li Mei'an, Wang Meng. Cloud task allocation strategy based on dynamic programming algorithm [J].Journal of Computer Applications, 2013, 33(SI): 20-25.

15. Sun Xiaobin, Xiao Renbin, Li Li. Explore on the Workload Model and the Total Consumption Model of the Planning of Concurrent Design [J]. China Mechanical Engineering, 1999(02): 207-210+243.

16. Guo Feng, Wu JianWei, Pan ShuangXia, Feng Peien, Chang Yan. Product development process modelling based on design structure matrix family [J]. Journal of Zhejiang University (Engineering Science), 2006(09): 1609-1613.

17. Yassine, Ali A. Whitney, Daniel E. Zambito, Tony. Assessment of rework probabilities for simulating product development processes using the Design Structure Matrix (DSM) [C]. Proceedings of the ASME Design Engineering Technical Conference, 2001(4): 105113.

18. Zhou Youjun, Cao Liang, Pan Yiqian. The optimal inventory model based on the dynamic programming method[C]. Proceedings of the 2 nd International Conference on Modelling and Simulation, ICMS2009, 2009(3): 74-78. 